\title{
A Rho-kinase inhibitor, fasudil, prevents development of diabetes and nephropathy in insulin-resistant diabetic rats
}

\author{
Yuichi Kikuchi, Muneharu Yamada, Toshihiko Imakiire, Taketoshi Kushiyama, Keishi Higashi, \\ Naomi Hyodo, Kojiro Yamamoto, Takashi Oda, Shigenobu Suzuki and Soichiro Miura \\ Department of Internal Medicine, National Defense Medical College, 3-2 Namiki, Tokorozawa, Saitama 359-8513, Japan \\ (Requests for offprints should be addressed to Y Kikuchi; Email: grd1615@ndmc.ac.jp)
}

\begin{abstract}
Fasudil, a Rho-kinase inhibitor, may improve insulin signaling. However, its long-term effect on metabolic abnormalities and its preventive effect on diabetic nephropathy are still unknown. We assessed these effects of fasudil in insulin-resistant diabetic rats, comparing them with those of an angiotensin II receptor blocker, olmesartan. Male Otsuka Long-Evans Tokushima fatty (OLETF) and Long-Evans Tokushima Otsuka, nondiabetic control, rats at 15 weeks of age were used. OLETF rats were randomized to receive a low or a high dose of fasudil or olmesartan for 25 weeks. To examine the therapeutic effects after the development of diabetes, OLETF rats at 30 weeks of age were given fasudil for 10 weeks. Administration of highdose fasudil completely suppressed the development of diabetes, obesity, and dyslipidemia and increased serum adiponectin levels in OLETF rats. High-dose olmesartan also
\end{abstract}

decreased hemoglobin A1c and increased serum adiponectin. There was a significant correlation between hemoglobin A1c and serum adiponectin or free fatty acid levels. The treatment with high-dose fasudil ameliorated proteinuria, glomerulosclerosis, renal interstitial fibrosis, and macrophage infiltration in OLETF rats. Olmesartan, even at the low dose, suppressed renal complications. The treatment with fasudil after the development of diabetes improved the metabolic abnormalities in OLETF rats, but could not suppress the progression of nephropathy. We conclude that the long-term treatment with fasudil prevents the development of diabetes, at least in part, by improving adipocyte differentiation in insulin-resistant diabetic rats. Early use of fasudil may prevent diabetic nephropathy.

Journal of Endocrinology (2007) 192, 595-603

\section{Introduction}

The population with type 2 diabetes mellitus and metabolic syndrome has been increasing worldwide and diabetic nephropathy is a leading cause in many countries, requiring renal replacement therapy. Obesity and insulin resistance are common characteristics in these conditions. To improve insulin resistance, biguanides and thiazolidinediones are widely used. Although these agents are very effective, serious adverse effects including lactic acidosis with biguanides, edema, body weight gain, heart failure, and liver dysfunction due to thiazolidinediones are well known. These facts indicate the need for a better therapeutic agent for type 2 diabetes.

A small GTP-binding protein, Rho, and its effector, Rhokinase, have several physiological and pathological functions, including stress fiber formation, cytokinesis, and cell migration, and contribute to calcium ion sensitivity in smooth muscle cells (Fukata et al. 2001). Rho/Rho-kinase participates in the regulation of systemic vascular tone and abnormal contraction of vascular smooth muscle, inducing hypertension and vasospasm of the coronary and cerebral arteries. Y-27632, a Rho-kinase inhibitor, dramatically reduced blood pressure in several hypertensive rats (Uehata et al. 1997). Another Rho-kinase inhibitor, fasudil, prolonged the maximum duration of exercise and time to the onset of ST segment depression in patients with stable effort angina (Shimokawa et al. 2002). In renal diseases, fasudil prevented hypertensive renal injuries by inhibiting expression of extracellular matrix genes, oxidative stress, cell proliferation, and macrophage infiltration in several hypertensive models (Kanda et al. 2003, Nishikimi et al. 2004, Ishikawa et al. 2006). Fasudil decreased urinary protein levels and reversed reduced nephrin expression in a puromycin aminonucleoside-induced nephrosis model (Shibata et al. 2006).

Insulin-stimulated GLUT4 translocation and glucose uptake in skeletal muscle are regulated by an insulin receptor/insulin receptor substrate (IRS)-1/phosphatidylinositol 3-kinase (PI3K)/Akt pathway (Kanai et al. 1993). A selective resistance to this pathway was demonstrated in the vascular tissues of insulin-resistant obese Zucker rats (Jiang et al. 1999). In vascular smooth muscle cells, activation of RhoA/Rho-kinase, ROK- $\alpha$, increased the association between Rho-kinase and IRS-1, leading to inhibition of insulin signaling (Begum et al. 2002). Recently, it was reported that treatment with fasudil for 4 weeks corrected the glucose and lipid metabolism in obese Zucker rats through 
the improvement in insulin signaling in skeletal muscles (Kanda et al. 2006). This finding may allow a new therapeutic strategy for the treatment of metabolic syndrome and type 2 diabetes mellitus. However, the long-term effect of Rhokinase inhibitors on metabolic abnormalities and its preventive effect on diabetic nephropathy are still unknown.

Based on these findings, we hypothesized that long-term administration of a Rho-kinase inhibitor might prevent the development and progression of diabetes and diabetic nephropathy in a spontaneous insulin-resistant diabetic model: Otsuka Long-Evans Tokushima fatty (OLETF) rats, which are obese, have hyperglycemia, hyperinsulinemia, hyperlipidemia, and hypertension (Kawano et al. 1992). First, fasudil was given to OLETF rats for 25 weeks from the early phase. Secondly, fasudil was given for 10 weeks after the development of diabetes to assess the therapeutic effects. In addition, these effects of fasudil were compared with those of an angiotensin II receptor blocker (ARB), for which the inhibition of the new development of diabetes (Lindholm et al. 2002, Julius et al. 2004, Yusuf et al. 2005) and renoprotective effects beyond lowering blood pressure (Brenner et al. 2001, Lewis et al. 2001, Viberti et al. 2002) have been proven in many clinical trials.

\section{Materials and Methods}

\section{Materials}

Male OLETF and Long-Evans Tokushima Otsuka (LETO) rats were obtained from Otsuka Pharmaceutical Tokushima Research Institute (Tokushima, Japan). Fasudil was obtained from Asahi Kasei Pharma Corporation (Tokyo, Japan) and olmesartan from Sankyo (Tokyo, Japan). The antibody against anti-rat ED1, which is a marker of monocytes/macrophages, was obtained from Cymbus Biotechnology (Chandlers Ford, Hants, UK); the antibody against phosphorylated myosin phosphatase target subunit (MYPT)-1, which is a marker of activation of the Rho/Rho-kinase pathway, from Upstate (Lake Placid, NY, USA); and the labeled streptavidin biotin (LSAB) kit from Dako (Carpinteria, CA, USA).

\section{Experimental protocols}

Protocol 1 Pathogen-free male OLETF and age-matched LETO, non-diabetic control, rats were used. Rats were given free access to tap water and standard rat chow. All studies followed the guidelines of National Defense Medical College for the care and use of laboratory animals in research. At 15 weeks of age, OLETF rats were divided into five groups: an untreated group, a low-dose fasudil $(30 \mathrm{mg} / \mathrm{kg})$ treatment group, a high-dose fasudil $(100 \mathrm{mg} / \mathrm{kg})$ treatment group, a low-dose olmesartan $(5 \mathrm{mg} / \mathrm{kg})$ treatment group, and a highdose olmesartan $(10 \mathrm{mg} / \mathrm{kg})$ treatment group $(n=7 \mathrm{in} \mathrm{each})$, matched for body weight (average 551.5 $\pm 10 \cdot 3 \mathrm{~g}$ ) and blood glucose (average 136.0 $\pm 5 \cdot 6 \mathrm{mg} / \mathrm{dl}$ ). Fasudil and olmesartan were dissolved in drinking water. These agents were given for 25 weeks. Laboratory data such as glucose, lipid and renal function, and renal pathological changes were compared among the six groups, including untreated LETO rats $(n=5)$. Systolic blood pressure was measured by the tail-cuff method and urine samples over $24 \mathrm{~h}$ were collected in metabolic cages at 40 weeks of age. After anesthesia by i.p. injection with sodium pentobarbital $(50 \mathrm{mg} / \mathrm{kg})$, blood was collected from the aorta between 0900 and $1000 \mathrm{~h}$ under non-fasting conditions and the kidneys were removed. One kidney was rapidly frozen in liquid nitrogen, and the other kidney was fixed in $10 \%$ formalin and embedded in paraffin.

Protocol 2 At 30 weeks of age, ten OLETF rats were divided into two groups: an untreated group $(n=5)$ and a high-dose fasudil $(100 \mathrm{mg} / \mathrm{kg})$ treatment group $(n=5)$, matched for body weight $(703 \cdot 6 \pm 12 \cdot 4$ vs $691 \cdot 4 \pm 29 \cdot 8 \mathrm{~g} ; P=0 \cdot 72)$ and blood glucose $(293 \cdot 6 \pm 35 \cdot 1$ vs $296 \cdot 8 \pm 46 \cdot 0 \mathrm{mg} / \mathrm{dl} ; P=0 \cdot 96)$. Fasudil was given for 10 weeks. The same parameters as Protocol 1 were compared among the three groups, including untreated LETO rats $(n=5)$. At the end of the study, the same procedures as Protocol 1 were performed.

\section{Biochemical assays}

Total blood glucose obtained from the tail vain was measured by the glucose oxidase method. Serum creatinine, total cholesterol, triglycerides, free fatty acid (FFA), and urinary creatinine and protein were measured by standard methods using an automatic analyzer, Hitachi 7180 (Hitachi). Creatinine clearance was calculated using the standard formula. Hemoglobin A1c levels were measured by the latex agglutination method. Serum adiponectin levels were determined by enzyme-linked immunosorbent assay (Otsuka, Tokyo, Japan).

\section{Renal histological study}

All formalin-fixed kidney sections $(3 \mu \mathrm{m})$ were stained with periodic acid-Schiff (PAS) and Masson's trichrome. Fifty full-sized glomeruli for each specimen were assessed on PAS-stained sections under a high-power field $(\times 400)$ and the levels of glomerulosclerosis in each glomerulus were semiquantitatively scored as follows: 0 , no sclerosis; 1 , sclerosis $<10 \%$ of glomeruli; 2 , sclerosis $10-25 \%$ of glomeruli; 3 , sclerosis $25-50 \%$ of glomeruli; 4 , sclerosis $>50 \%$ of glomeruli. To evaluate interstitial fibrosis, 20 fields for each section were assessed on Masson's trichrome-stained sections $(\times 200)$. Semiquantitative analysis in each field was assessed as follows: 0 , no fibrosis; 1 , fibrosis $<10 \%$ of areas; 2 , fibrosis $10-25 \%$ of areas; 3 , fibrosis $25-50 \%$ of areas; 4 , fibrosis $>50 \%$ of areas. The averages of the glomerulosclerosis and interstitial fibrosis scores were calculated from the total evaluated glomeruli or interstitial lesions in each section. These microscopic evaluations were performed by two observers without prior prejudicial information. 


\section{Immunohistochemistry}

Three micrometer thick formalin-fixed sections were used to detect ED1-positive cells in the kidney. Deparaffinized sections were treated with proteinase $\mathrm{K}$ for $7 \mathrm{~min}$ for optimal antigen retrieval and incubated with $3 \% \mathrm{H}_{2} \mathrm{O}_{2}$ for $10 \mathrm{~min}$ to block endogenous peroxidase activity. After blocking with $7 \%$ bovine serum albumin in PBS, the sections were incubated with a primary antibody for ED1 $(\times 200)$ for $60 \mathrm{~min}$ at $37^{\circ} \mathrm{C}$. Thereafter, the sections were incubated with
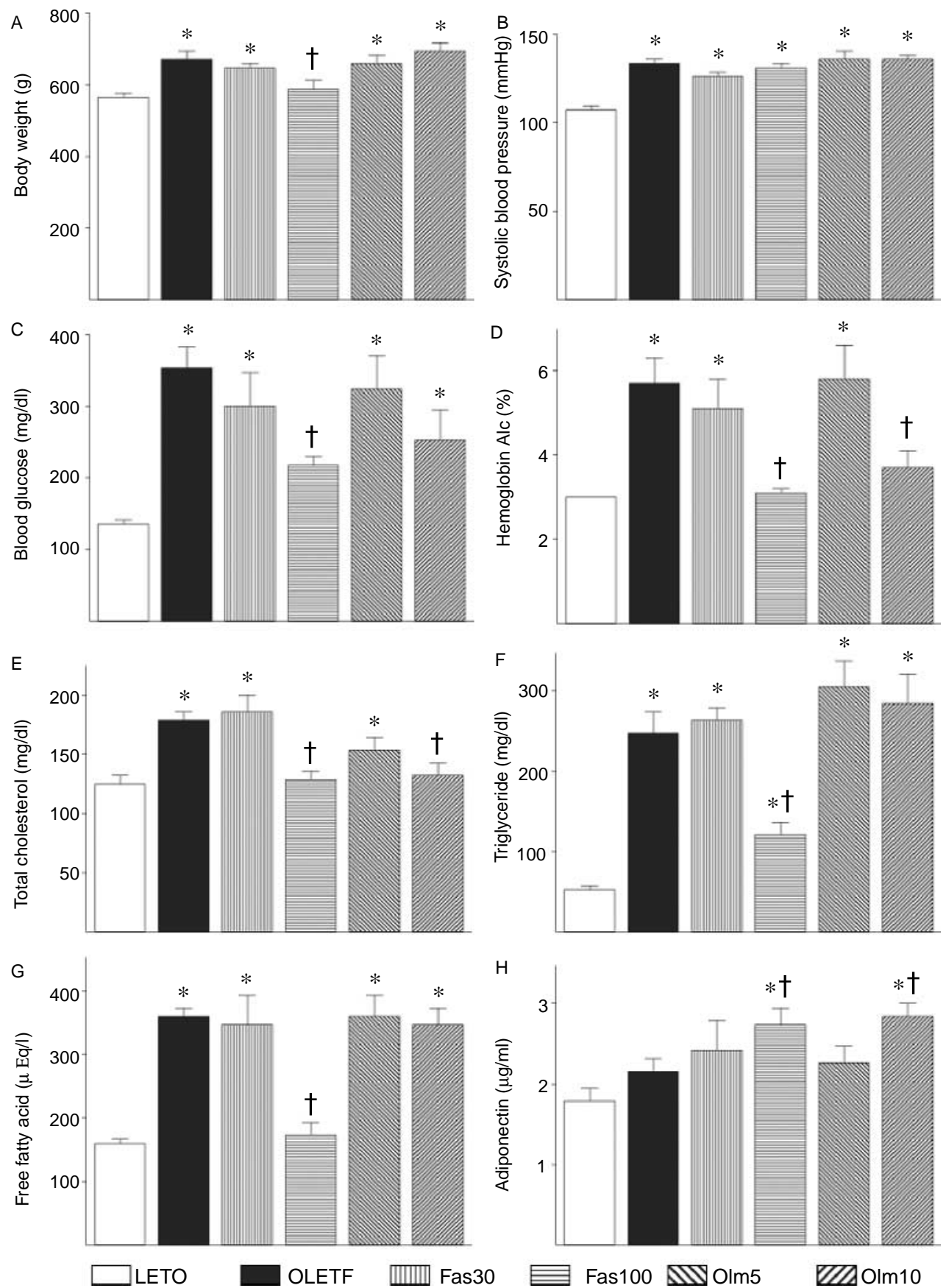

Figure 1 Body weight (A), blood pressure (B), glucose (C), hemoglobin A1c (D), and lipid metabolism (E-H) in OLETF rats treated with fasudil or olmesartan in Protocol $1(n=5-7)$. Fas30 or Fas100, OLETF rats treated with 30 or $100 \mathrm{mg} / \mathrm{kg}$ fasudil dissolved in drinking water; Olm5 or Olm10, OLETF rats treated with 5 or $10 \mathrm{mg} / \mathrm{kg}$ olmesartan dissolved in drinking water. ${ }^{*} P<0 \cdot 05$ vs LETO rats, ${ }^{+} P<0 \cdot 05$ versus untreated OLETF rats. 
a DAKO LSAB system link antibody for $10 \mathrm{~min}$, followed by incubation with peroxidase-conjugated streptavidin for $10 \mathrm{~min}$ at room temperature. After washing with PBS, the sections were stained with a $3,3^{\prime}$-diaminobenzidine solution and then counterstained with hematoxylin. More than 20 non-overlapping areas for each section were assessed under high-power magnification $(\times 400)$, and the number of ED1-positive cells in each area was counted and averaged in each group.

\section{Western blot analysis}

To assess activation of Rho/Rho-kinase pathway in LETO and OLETF rats, western blot analysis of phosphorylated MYPT-1 was performed. The samples for western blotting were prepared from the renal cortex of LETO and OLETF rats. Protein was extracted from each renal sample by homogenizing it in a lysis buffer. The composition of the lysis buffer was $20 \mathrm{mM}$ Tris-HCl (pH 7·5), $150 \mathrm{mM} \mathrm{NaCl}$, $1 \mathrm{mM}$ EDTA, $1 \mathrm{mM}$ EGTA, $1 \mathrm{mM}$ sodium vanadate, 1\% NP40, and a protease inhibitor cocktail (Sigma). Protein concentration was assayed by the Bio-Rad protein assay reagent, and $50 \mu \mathrm{g}$ protein were applied to each well. Samples were denatured for $3 \mathrm{~min}$ at $98{ }^{\circ} \mathrm{C}$, electrophoresed on a gradient (4-15\%) polyacrylamide gel (Bio-Rad Laboratories), and electrophoretically transferred onto nitrocellulose membranes. After blocking with a blocking buffer (Pierce, Rockford, IL, USA), the membrane was incubated with a primary antibody for phosphorylated MYPT-1 $(\times 500)$ overnight at $4{ }^{\circ} \mathrm{C}$. After washing with Tris-buffered saline containing $0 \cdot 05 \%$ Tween 20 , horseradish peroxidase-linked anti-rabbit $\operatorname{IgG}(\times 2000)$ was incubated for $1 \mathrm{~h}$ at room temperature. The specific protein expression was visualized using enhanced chemiluminescence western blotting detection reagents (Amersham). The phosphorylated MYPT-1 protein levels were corrected by the $\beta$-actin protein levels.

\section{Statistical analysis}

Results are expressed as the mean \pm s.E.M. Statistical analyses were performed using analysis of variance followed by Fisher's multiple comparisons. Correlations between several parameters were assessed by Spearman's correlation test. A $P$ value of $<0 \cdot 05$ was considered significant.

\section{Results}

Effects of fasudil and olmesartan on metabolic abnormalities in OLETF rats

The body weights of OLETF rats at 40 weeks of age were significantly higher than those of LETO rats (Fig. 1A). Systolic blood pressure in OLETF rats was higher than that of LETO rats (Fig. 1B). OLETF rats had significantly increased blood glucose, hemoglobin A1c, serum total cholesterol, triglycerides, and FFA levels compared with those of LETO rats (Fig. 1C-G). Serum adiponectin levels showed no significant difference between OLETF and LETO rats (Fig. 1H). Administration of low-dose fasudil did not affect these parameters in OLETF rats. The body weights of OLETF rats treated with high-dose fasudil were not different from those of LETO rats (Fig. 1A). High-dose fasudil did not change systolic blood pressure in OLETF rats (Fig. 1B), as observed in the previous reports (Nishikimi et al. 2004, Ishikawa et al. 2006). Administration of high-dose fasudil completely suppressed the development of diabetes and dyslipidemia in OLETF rats (Fig. 1C-G). Serum adiponectin levels in OLETF rats treated with high-dose fasudil were significantly higher compared with those in LETO or untreated OLETF rats (Fig. 1H). Olmesartan did not affect the body weights or blood pressure in OLETF rats (Fig. 1A and B). The treatment with high-dose olmesartan in OLETF rats significantly decreased hemoglobin A1c and total cholesterol levels, as well as increased serum adiponectin levels compared with untreated OLETF rats (Fig. 1D, E and $\mathrm{H}$ ). However, high-dose olmesartan did not improve serum triglycerides or FFA levels (Fig. 1F and G).

In OLETF rats, serum adiponectin and FFA levels, which are associated with adipocyte differentiation, were significantly correlated with blood glucose, hemoglobin A1c, and total cholesterol levels (Table 1).

\section{Phosphorylated MYPT-1 levels in the renal cortex of OLETF rats}

Phosphorylated MYPT-1 protein levels in the renal cortex of OLETF rats at 40 weeks of age were significantly higher than those of LETO rats (Fig. 2). High-dose fasudil significantly suppressed the phosphorylated MYPT-1 levels in OLETF rats. Low-dose fasudil and olmesartan (both doses) did not affect the phosphorylated MYPT-1 levels.

Table 1 Correlation between adiponectin or free fatty acid and blood glucose, hemoglobin A1c, or lipids in OLETF rats

\begin{tabular}{|c|c|c|c|c|c|}
\hline & Blood glucose & Hemoglobin A1c & Total cholesterol & Triglyceride & Free fatty acid \\
\hline Adiponectin & $\begin{array}{l}r=-0.748 \\
P<0.001\end{array}$ & $\begin{array}{l}r=-0.822 \\
P<0 \cdot 001\end{array}$ & $\begin{array}{l}r=-0.594 \\
P=0.001\end{array}$ & NS & NS \\
\hline Free fatty acid & $\begin{array}{l}r=0.544 \\
P=0.002\end{array}$ & $\begin{array}{l}r=0.528 \\
P=0.002\end{array}$ & $\begin{array}{l}r=0.589 \\
P<0.001\end{array}$ & $\begin{array}{l}r=0.561 \\
P=0.001\end{array}$ & - \\
\hline
\end{tabular}

NS; not significant. 


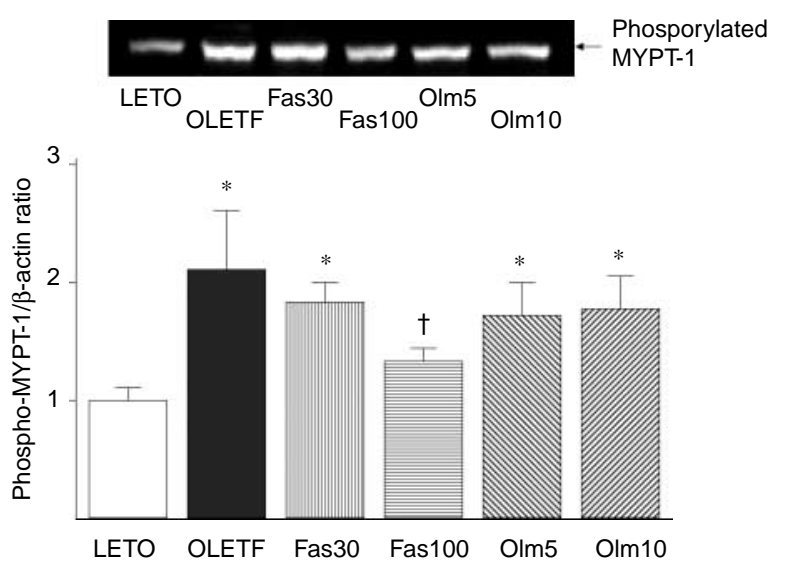

Figure 2 Phosphorylated MYPT-1 protein expression in the renal cortex of LETO and OLETF rats in Protocol 1. The photograph shows the representative results of western blotting for phosphorylated MYPT-1 (130 kDa). The bar graph shows the abundance of phosphorylated MYPT-1 protein expression relative to $\beta$-actin. ${ }^{*} P<0 \cdot 05$ vs LETO rats, ${ }^{\dagger} P<0 \cdot 05$ vs untreated OLETF rats.

Effects of fasudil and olmesartan on renal complications in OLETF rats

Creatinine clearance did not show significant differences among the groups (Fig. 3A). Urinary protein levels of OLETF rats were significantly higher than those of LETO rats (Fig. 3B). High-dose fasudil and both doses of olmesartan significantly suppressed the excretion of urinary protein.

Glomerular lesions in OLETF rats were characterized by hyalinosis, thickening of the basement membrane, mesangial expansion, and sclerotic lesions (Fig. 4A). Glomerulosclerosis and the interstitial fibrosis score in OLETF rats were significantly higher than those of LETO rats (Fig. 4B and C). The number of ED1-positive cells in the kidneys was significantly increased in OLETF rats (Fig. 4D). These pathological changes and ED1-positive cell infiltration were suppressed by the treatments with high-dose fasudil and both doses of olmesartan (Fig. 4A-D). Low-dose fasudil also suppressed the interstitial fibrosis score and the number of ED1-positive cells compared with untreated OLETF rats.

\section{Effects offasudil after the development of diabetes in OLETF rats}

Administration of high-dose fasudil for 10 weeks after the development of diabetes did not affect the body weight $(664 \cdot 8 \pm 41.5$ vs $596 \cdot 6 \pm 42 \cdot 4 \mathrm{~g} ; P=0 \cdot 19)$ or the systolic blood pressure $(138.5 \pm 3.4$ vs $141 \cdot 1 \pm 2.1 \mathrm{mmHg} ; P=0.54)$ in OLETF rats. The blood glucose and hemoglobin A1c levels in OLETF rats treated with fasudil were significantly lower than those in untreated OLETF rats (Fig. 5A and B). With short-term treatment with fasudil, serum total cholesterol, triglycerides, and FFA also significantly decreased and serum adiponectin increased in OLETF rats (Fig. 5C-F). Urinary protein levels, glomerulosclerosis, and interstitial fibrosis scores did not change after treatment with fasudil (Fig. 6A-C).

\section{Discussion}

In this study, we demonstrated that administration of a Rho-kinase inhibitor, fasudil, prevented the development and progression of obesity, hyperglycemia, dyslipidemia, and nephropathy in diabetic OLETF rats. The improvement in blood glucose and hemoglobin A1c levels was strongly correlated with serum adiponectin and FFA levels in OLETF rats.

Adipose tissue, especially visceral fat, expresses numerous genes and secretes biologically active molecules, called 'adipocytokines' (Maeda et al. 1997, Funahashi et al. 1999). Among them, leptin, resistin, tumor necrosis factor (TNF)- $\alpha$, and interleukin- 6 are proinflammatory adipocytokines, while adiponectin is an anti-inflammatory adipocytokine. An imbalance in them leads to the development of atherosclerosis and insulin resistance (Axelsson et al. 2005). Since higher concentrations of adiponectin protected against later development of type 2 diabetes in humans (Lindsay et al. 2002), adiponectin has been thought to play a key role in the development of type 2 diabetes. Fasudil significantly increased
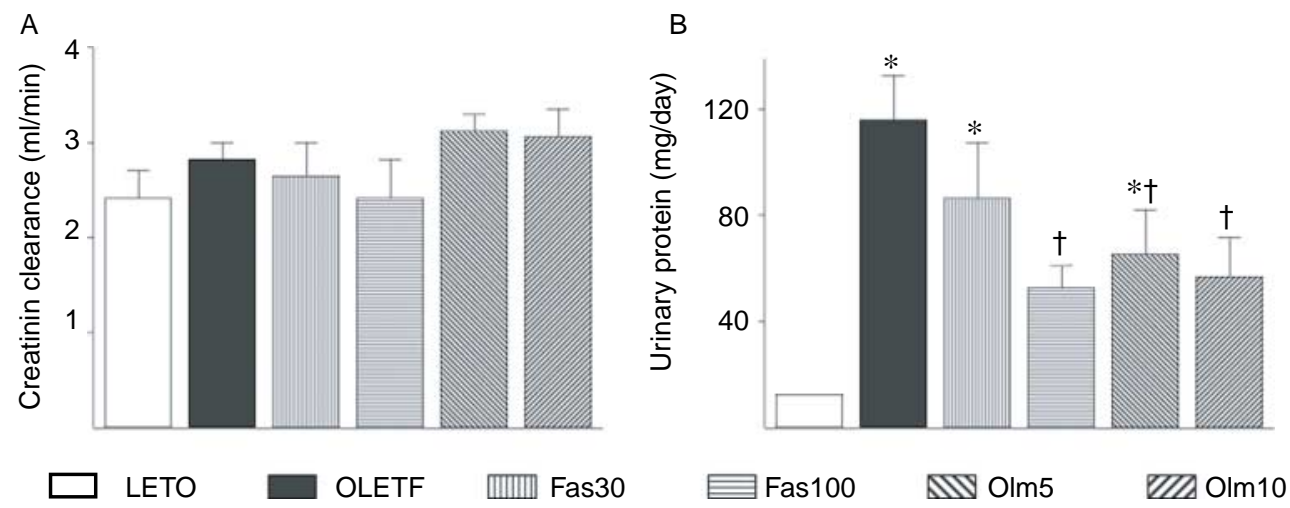

Figure 3 Creatinine clearance $(A)$ and urinary protein $(B)$ in OLETF rats treated with fasudil or olmesartan in Protocol $1 .{ }^{*} P<0.05$ vs LETO rats, ${ }^{\dagger} P<0.05$ vs untreated OLETF rats. 
serum adiponectin levels in OLETF rats. Peroxisome proliferator-activated receptor (PPAR) $\gamma$ regulates adipocyte gene expression and differentiation. Thiazolidinediones, specific ligands for PPAR- $\gamma$, also increased the plasma adiponectin levels in insulin-resistant humans and animals, as well as improving hyperglycemia (Maeda et al. 2001, Choi et al. 2005). Pioglitazone, a thiazolidinedione, suppressed the Rho/Rho-kinase pathway in aortic tissues from spontaneously hypertensive rats (Wakino et al. 2004). Both fasudil and thiazolidinediones might exert a beneficial effect

\section{A}

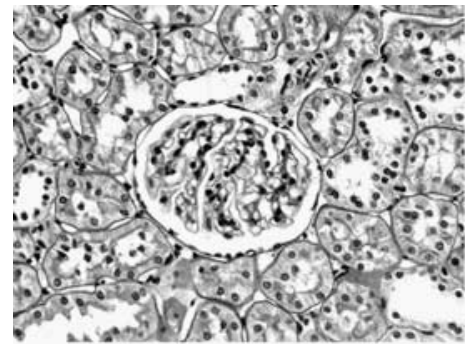

LETO

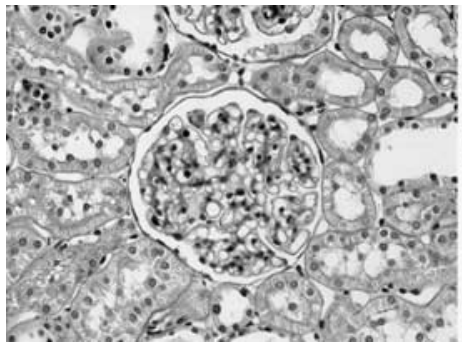

Fas 100
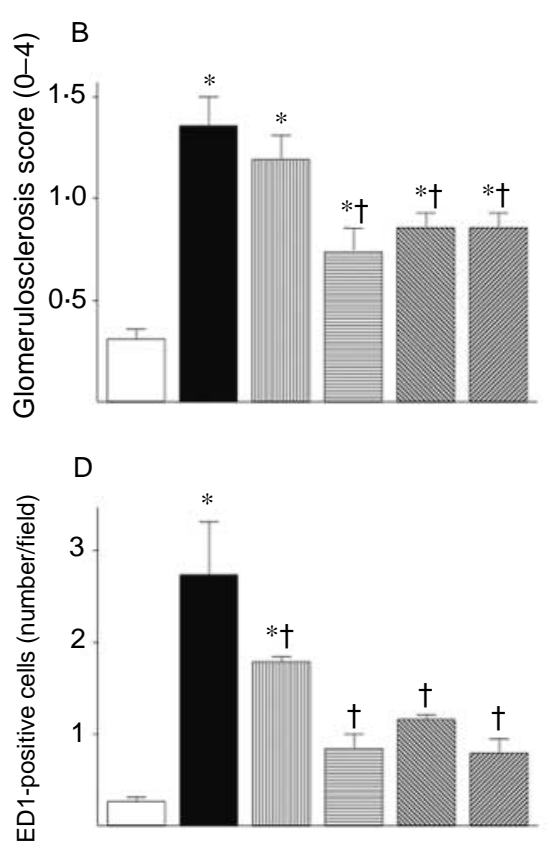

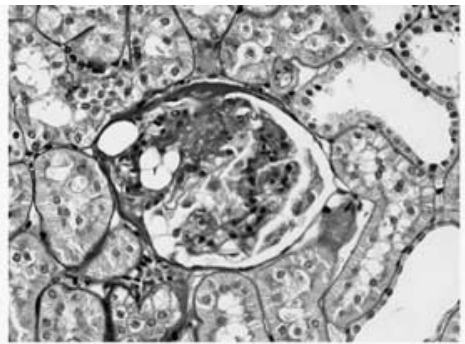

OLETF

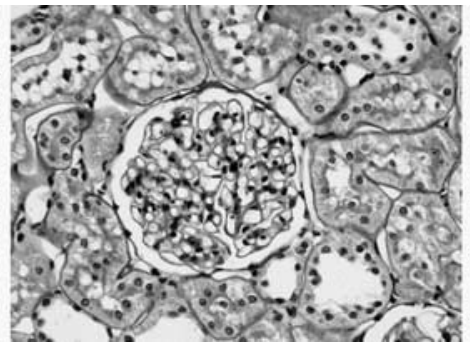

Olm5

C

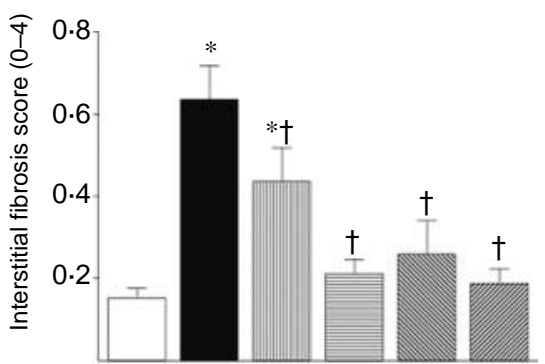

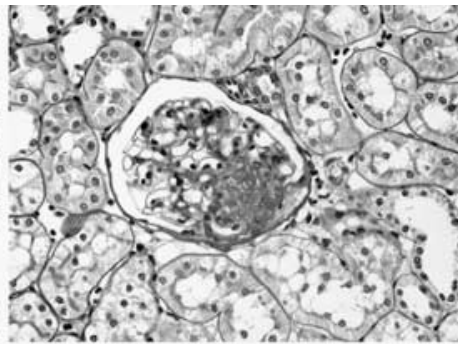

Fas30

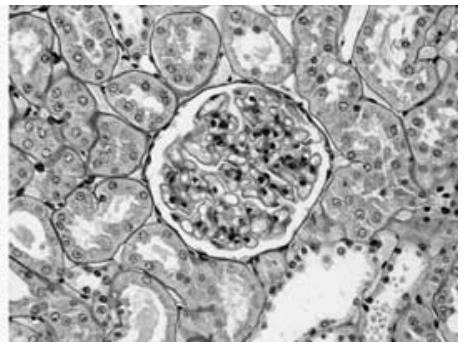

Olm10 
A

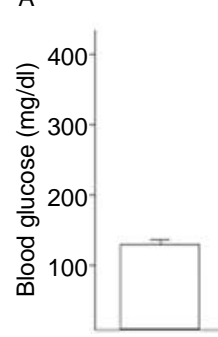

D
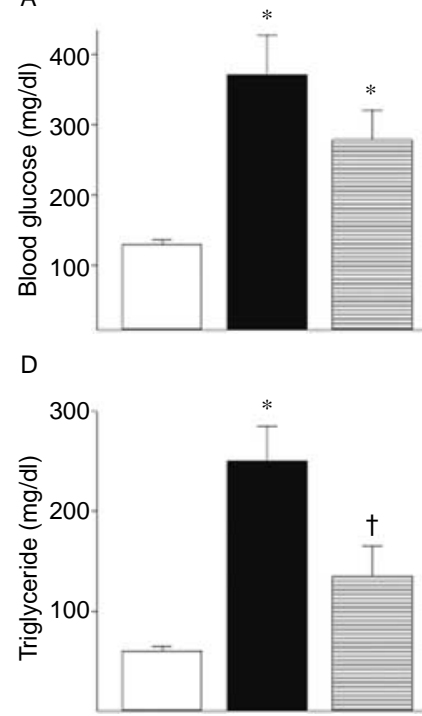

B

$\mathrm{E}$

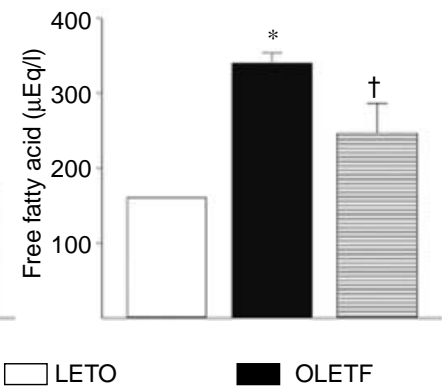

LETO

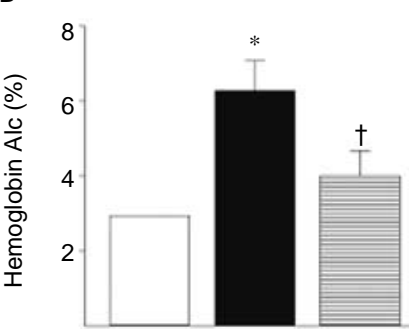

C

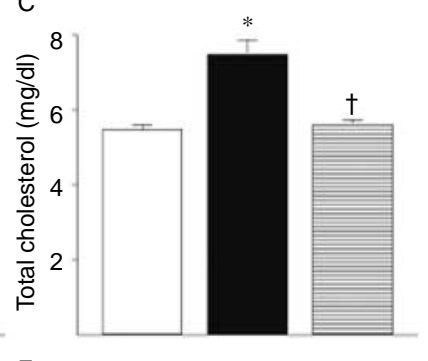

$\mathrm{F}$

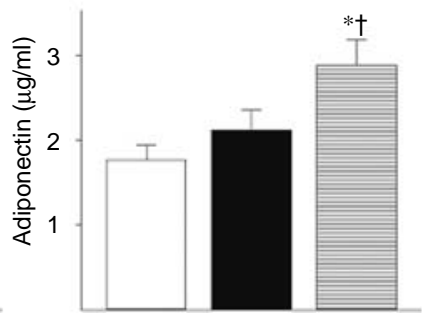

Fas 100

Figure 5 Glucose and hemoglobin Alc (A and B) and lipid metabolism (C-F) in OLETF rats treated with highdose fasudil for 10 weeks after the development of diabetes in Protocol $2(n=5)$. Fas100, OLETF rats treated with $100 \mathrm{mg} / \mathrm{kg}$ fasudil dissolved in drinking water. ${ }^{*} P<0.05$ vs LETO rats, ${ }^{+} P<0 \cdot 05$ vs untreated OLETF rats.

on glucose and lipid metabolism, at least in part, through the inhibition of Rho/Rho-kinase activation. Adipocyte hypertrophy induces lipolysis and increases secretion of FFA. The increased levels of FFA might mean an abnormality in adipocyte differentiation. Long-chain fatty acyl-CoA is the activated form of intracellular FFA. Diacylglycerol is generated through esterification of fatty acyl-CoA to glycerol-3-phosphate and activates protein kinase C (PKC). Activated PKC induced insulin resistance by promoting serine phosphorylation of IRS-1 (Hegarty et al. 2003). A high plasma concentration of FFA is found in patients with type 2 diabetes. In the present study, serum FFA levels in OLETF rats were significantly higher than those in LETO rats. Administration of high-dose fasudil completely suppressed serum FFA levels in OLETF rats. Kanda et al. (2006) reported that fasudil improved glucose metabolism by inhibiting serine phosphorylation of IRS-1 and the augmentation of insulin signaling in the skeletal muscles of obese Zucker rats. Our results suggest that fasudil might improve metabolic abnormalities not only by improving insulin signaling, but also by correcting adipocyte differentiation.

Olmesartan also improved hemoglobin $\mathrm{A} 1 \mathrm{c}$ and serum total cholesterol levels, and increased serum adiponectin levels in OLETF rats. The protective effects of ARB against the development of diabetes have been proven in many clinical trials (Lindholm et al. 2002, Julius et al. 2004, Yusuf et al. 2005). Angiotensin II was reported to activate Rho-kinase in an angiotensin II-induced cardiovascular hypertrophy model (Higashi et al. 2003). Therefore, we hypothesized that the effects of ARB on metabolic abnormalities in insulin-resistant models might be due to the inhibition of Rho-kinase activation. However, the activation of Rho-kinase, shown by

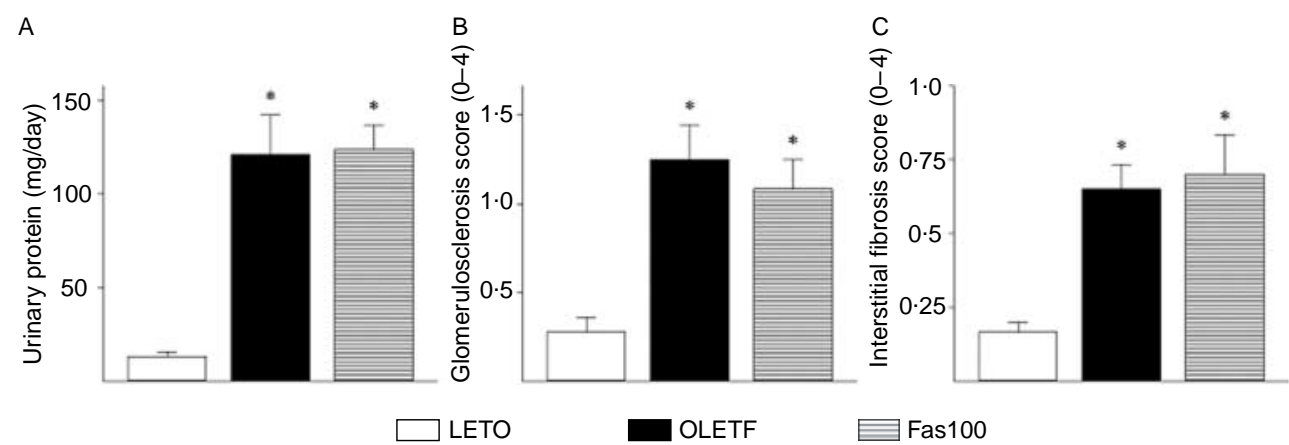

Figure 6 Urinary protein $(A)$ and renal histological changes (B and C) in OLETF rats treated with fasudil $\left(100 \mathrm{mg} / \mathrm{kg}\right.$ dissolved in drinking water) for 10 weeks in Protocol 2. ${ }^{*} P<0 \cdot 05$ vs LETO rats. 
phosphorylated MYPT-1 levels, in OLETF rats could not be suppressed by olmesartan. Valsartan, another ARB, improved glucose tolerance through an increase in tyrosine phosphorylation of IRS-1, activation of PI3K, and translocation of GLUT4 to the plasma membrane in skeletal muscle in type 2 diabetic KK-Ay mice (Shiuchi et al. 2004). Olmesartan might improve the insulin resistance of OLETF rats by the same mechanisms as valsartan. Olmesartan significantly increased serum adiponectin levels in OLETF rats, but could not affect serum triglycerides or FFA levels. Angiotensin II infusion decreased plasma adiponectin levels in rats and olmesartan ameliorated this hypoadiponectinemia ( $\mathrm{R}$ an et al. 2006). However, angiotensin II infusion did not affect plasma levels of the other adipocytokines such as leptin or TNF- $\alpha$. These findings suggest that olmesartan did not improve the adipocyte differentiation itself, although the exact mechanism of increased serum adiponectin by olmesartan remains unknown. Summarizing the above findings, fasudil improved glucose and lipid abnormalities in insulin-resistant models by improving both adipocyte differentiation and insulin signaling in skeletal muscle, more effectively than ARB. The finding that short-term administration of fasudil after the development of diabetes significantly ameliorated the metabolic abnormalities in OLETF rats was notable.

High-dose fasudil and olmesartan inhibited the progression of diabetic nephropathy without affecting blood pressure. In several hypertensive rats, fasudil inhibited the expression of extracellular matrix genes, such as transforming growth factor- $\beta$, collagens I and III, oxidative stress, cell proliferation and macrophage infiltration in the kidneys and ameliorated urinary protein, glomerulosclerosis, and tubulointerstitial changes (Kanda et al. 2003, Nishikimi et al. 2004, Ishikawa et al. 2006). Since these changes are also found in diabetic nephropathy, fasudil was expected to have beneficial effects on diabetic nephropathy. In OLETF rats, high-dose fasudil significantly suppressed the urinary protein, glomerulosclerosis, interstitial fibrosis, and macrophage infiltration in the kidneys. However, it was unclear whether these effects were independent of the improvement in metabolic abnormalities. Low-dose fasudil, which could not affect glucose and lipid metabolism, decreased urinary protein by $25 \%$. Glomerulosclerosis score did not change, but the interstitial fibrosis score and macrophage infiltration were significantly inhibited by low-dose fasudil. These results suggest that fasudil may have renoprotective effects in diabetic nephropathy beyond its effects on metabolism in OLETF rats. Our previous study showed that macrophage infiltration in the kidneys was an important event in the development of diabetic nephropathy and was correlated with the pathological changes in OLETF rats (Kikuchi et al. 2005). The improvement in interstitial fibrosis might be due to the inhibition of cell migration by fasudil. It was reported that fasudil suppressed renal interstitial fibrosis by inhibiting the macrophage infiltration in rat kidneys with unilateral ureteral obstruction (Satoh et al. 2002). In this report, the migration of monocytes in vitro was dose dependently inhibited by fasudil. However, the therapeutic effects of high-dose fasudil initiated in a later stage of diabetic nephropathy were not demonstrated in our study. An additional study using different models may be required to resolve this issue.

In conclusion, long-term treatment with fasudil prevents the development of diabetes and dyslipidemia, at least in part, through the improvement in adipocyte differentiation in insulin-resistant diabetic rats. The use of fasudil from the early phase may prevent diabetic nephropathy. In addition to thiazolidinediones and biguanides, fasudil may become a new therapeutic strategy for insulin resistance, which is characteristic of metabolic syndrome and type 2 diabetes.

\section{Acknowledgements}

The authors declare that there is no conflict of interest that would prejudice the impartiality of this scientific work.

\section{References}

Axelsson J, Heimburger O, Lindholm B \& Stenvinkel P 2005 Adipose tissue and its relation to inflammation: the role of adipokines. Journal of Renal Nutrition 15 131-136.

Begum N, Sandu OA, Ito M, Lohmann SM \& Smolenski A 2002 Active Rho kinase $(\mathrm{ROK}-\alpha)$ associates with insulin receptor substrate-1 and inhibits signaling in vascular smooth muscle cells. Journal of Biological Chemistry 277 6214-6222.

Brenner BM, Cooper ME, de Zeeuw D, Keane WF, Mitch WE, Parving HH, Remuzzi G, Snapinn SM, Zhang Z, Shahinfar S et al. 2001 Effects of losartan on renal and cardiovascular outcomes in patients with type 2 diabetes and nephropathy. New England Journal of Medicine 345 861-869.

Choi KC, Ryu OH, Lee KW, Kim JA, Seo JA, Kim SG, Kim NH, Choi DS, Baik SH \& Choi KM 2005 Effect of PPAR $-\alpha$ and $-\gamma$ agonist on the expression of visfatin, adiponectin, and TNF- $\alpha$ in visceral fat of OLETF rats. Biochemical and Biophysical Research Communications 336 747-753.

Fukata Y, Amano M \& Kaibuchi K 2001 Rho-Rho-kinase pathway in smooth muscle contraction and cytoskeletal reorganization of non-muscle cells. Trends in Pharmacological Sciences 22 32-39.

Funahashi T, Nakamura T, Shimomura I, Maeda K, Kuriyama H, Takahashi M, Arita Y, Kihara S \& Matsuzawa Y 1999 Role of adipocytokines on the pathogenesis of atherosclerosis in visceral obesity. Internal Medicine 38 202-206.

Hegarty BD, Furler SM, Ye J, Cooney GJ \& Kraegen EW 2003 The role of intramuscular lipid in insulin resistance. Acta Physiologica Scandinavica 178 373-383.

Higashi M, Shimokawa H, Hattori T, Hiroki J, Mukai Y, Morikawa K, Ichiki T, Takahashi S \& Takeshita A 2003 Long-term inhibition of Rho-kinase suppresses angiotensin II-induced cardiovascular hypertrophy in rats in vivo. Circulation Research 93 767-775.

Ishikawa Y, Nishikimi T, Akimoto K, Ishimura K, Ono H \& Matsuoka H 2006 Long-term administration of Rho-kinase inhibitor ameliorates ranal damage in malignant hypertensive rats. Hypertension 47 1075-1083.

Jiang ZY, Lin YW, Clemont A, Feener EP, Hein KD, Igarashi M, Yamauchi T, White MF \& King GL 1999 Characterization of selective resistance to insulin signaling in the vasculature of obese Zucker $(\mathrm{fa} / \mathrm{fa})$ rats. Journal of Clinical Investigation 104 447-457.

Julius S, Kjeldsen SE, Weber M, Brunner HR, Ekman S, Hansson L, Hua T, Laragh J, McInnes GT, Mitchell L et al. 2004 Outcomes in hypertensive patients at high cardiovascular risk treated with regimens based on valsartan or amlodipine: the VALUE randomised trial. Lancet 363 2022-2031. 
Kanai F, Ito K, Todaka M, Hayashi H, Kamohara S, Ishii K, Okada T, Hazeki O, Ui M \& Ebina Y 1993 Insulin-stimulated GLUT4 translocation is relevant to the phosphorylation of IRS-1 and the activity of PI3-kinase. Biochemical and Biophysical Research Communications 195 762-768.

Kanda T, Wakino S, Hayashi K, Homma K, Ozawa Y \& Saruta T 2003 Effect of fasudil on Rho-kinase and nephropathy in subtotally nephrectomized spontaneously hypertensive rats. Kidney International 64 2009-2019.

Kanda T, Wakino S, Homma K, Yoshioka K, Takematsu S, Hasegawa K, Takamatsu I, Sugano N, Hayashi K \& Saruta T 2006 Rho-kinase as a molecular target for insulin resistance and hypertension. FASEB Journal 20 169-171.

Kawano K, Hirashima T, Mori S, Saitoh Y, Kurosumi M \& Natori T 1992 Spontaneous long-term hyperglycemic rat with diabetic complications. Otsuka Long-Evans Tokushima Fatty (OLETF) strain. Diabetes 41 1422-1428.

Kikuchi Y, Imakiire T, Yamada M, Saigusa T, Hyodo T, Hyodo N, Suzuki S \& Miura S 2005 Mizoribine reduces renal injury and macrophage infiltration in non-insulin-dependent diabetic rats. Nephrology, Dialysis, Transplantation 20 1573-1581.

Lewis EJ, Hunsicker LG, Clarke WR, Berl T, Pohl MA, Lewis JB, Ritz E, Atkins RC, Rohde R, Raz I et al. 2001 Renoprotective effect of the angiotensin-receptor antagonist irbesartan in patients with nephropathy due to type 2 diabetes. New England Journal of Medicine 345 851-860.

Lindholm LH, Ibsen H, Borch-Johnsen K, Olsen MH, Wachtell K, Dahlof B, Devereux RB, Beevers G, de Faire U, Fyhrquist F et al. 2002 Risk of newonset diabetes in the Losartan Intervention For Endpoint reduction in hypertension study. Journal of Hypertension 20 1879-1886.

Lindsay RS, Funahashi T, Hanson RL, Matsuzawa Y, Tanaka S, Tataranni PA, Knowler WC \& Krakoff J 2002 Adiponectin and development of type 2 diabetes in the Pima Indian population. Lancet $36057-58$.

Maeda K, Okubo K, Shimomura I, Mizuno K, Matsuzawa Y \& Matsubara K 1997 Analysis of an expression profile of genes in the human adipose tissue. Gene 190 227-235.

Maeda N, Takahashi M, Funahashi T, Kihara S, Nishizawa H, Kishida K, Nagaretani H, Matsuda M, Komuro R, Ouchi N et al. 2001 PPAR $\gamma$ ligands increase expression and plasma concentration of adiponectin, an adiposederived protein. Diabetes 50 2094-2099.

Nishikimi T, Akimoto K, Wang X, Mori Y, Tadokoro K, Ishikawa Y, Shimokawa H, Ono H \& Matsuoka H 2004 Fasudil, a Rho-kinase inhibitor, attenuates glomerulosclerosis in Dahl salt-sensitive rats. Journal of Hypertension 22 1787-1796.

Ran J, Hirano T, Fukui T, Saito K, Kageyama H, Okada K \& Adachi M 2006 Angiotensin II infusion decreases plasma adiponectin level via its type 1 receptor in rats: an implication for hypertension-related insulin resistance. Metabolism 55 478-488.
Satoh S, Yamaguchi T, Hitomi A, Ato N, Shiraiwa K, Ikegaki I, Asano T \& Shimokawa H 2002 Fasudil attenuates interstitial fibrosis in rat kidneys with unilateral uretral obstruction. European Journal of Pharmacology $\mathbf{4 5 5}$ 169-174.

Shibata S, Nagase M \& Fujita T 2006 Fluvastatin ameliorates podocyte injury in proteinuric rats via modulation of excessive Rho signaling. Journal of the American Society of Nephrology 17 754-764.

Shimokawa H, Hiramori K, Iinuma H, Hosoda S, Kishida H, Osada H, Katagiri T, Yamauchi K, Yui Y, Minamino T et al. 2002 Anti-anginal effect of fasudil, a Rho-kinase inhibitor, in patients with stable effort angina: a multicenter study. Journal of Cardiovascular Pharmacology 40 751-761.

Shiuchi T, Iwai M, Li HS, Wu L, Min LJ, Li JM, Okumura M, Cui TX \& Horiuchi M 2004 Angiotensin II type-1 receptor blocker valsartan enhances insulin sensitivity in skeletal muscles of diabetic mice. Hypertension 43 1003-1010.

Uehata M, Ishizaki T, Satoh H, Ono T, Kawahara T, Morishita T, Tamakawa H, Yamagami K, Inui J, Maekawa M et al. 1997 Calcium sensitization of smooth muscle mediated by a Rho-associated protein kinase in hypertension. Nature 389 990-994.

Viberti G, Wheeldon NM \& MicroAlbuminuria Reduction With VALsartan (MARVAL) Study Investigators 2002 Microalbuminuria reduction with valsartan in patients with type 2 diabetes mellitus: a blood pressureindependent effect. Circulation 106 672-678.

Wakino S, Hayashi K, Kanda T, Takematsu S, Homma K, Yoshioka K, Takamatsu I \& Saruta T 2004 Peroxisome proliferator-activated receptor $\gamma$ ligands inhibit Rho/Rho kinase pathway by inducing protein tyrosine phosphatase SHP-2. Circulation Research 95 e45-e55.

Yusuf S, Ostergren JB, Gerstein HC, Pfeffer MA, Swedberg K, Granger CB, Olofsson B, Probstfield J, McMurray JV \& Candesartan in Heart FailureAssessment of Reduction in Mortality and Morbidity Program (CHARM) Investigators 2005 Effects of candesartan on the development of a new diagnosis of diabetes mellitus in patients with heart failure. Circulation $\mathbf{1 1 2}$ $48-53$.

Received in final form 2 December 2006 Accepted 6 December 2006 Made available online as an Accepted Preprint 28 December 2006 